\title{
Influence of ultrasound treatment and dynamic (in-situ) ultrasound loading on the temperature hysteresis of electrophysical characteristics in irradiated $\boldsymbol{n}$-Si-Fz
}

\author{
V.M. Babych, Ja.M. Olikh, M.D. Tymochko* \\ V. Lashkaryov Institute of Semiconductor Physics, NAS of Ukraine \\ 41, prospect Nauky, 03028 Kyiv, Ukraine \\ *Corresponding author phone: 38 (044) 525-62-56, e-mail: tymochko@ukr.net
}

\begin{abstract}
Presented in this paper are experimental results of ultrasound treatment (UST) and dynamic ultrasound loading (USL) influences on the electric activity of radiation defects (after $\gamma$-irradiation) in crystals $n$-Si-Fz. The results are obtained using the Hall effect method within the temperature range 100-300 K. Peculiarities of US action in the treatment and loading modes on the temperature hysteresis of electrophysical characteristics in investigated material (extension and narrowing) were analyzed. Diffusion and deformation mechanisms responsible for US modification of defect complexes have been suggested.
\end{abstract}

Keywords: ultrasound treatment, ultrasound (in-situ) loading, irradiation defects, electric activity, silicon, Hall effect.

Manuscript received 15.08.09; accepted for publication 10.09.09; published online 30.10.09.

\section{Introduction}

Nowadays, radiation defects (RD) in silicon crystals induced by various kinds of irradiation are well known, but physics of ultrasound (US) interactions with these defects, their sensitivity to acoustic vibrations are not sufficiently investigated. In previous papers, the influence of ultrasonic treatment on the diffusion length of minority charge carriers in $p$-type silicon crystals [1], on the lifetime of non-equilibrium charge carriers [2] and on the intrinsic friction [3] in $\gamma$-irradiated silicon samples was studied.

In the course of investigations of the US influence on the electro-physical (EPh) characteristics of $\gamma$ irradiated silicon samples, it has been found that separate $\mathrm{RD}-A$-centres $\left(E_{\mathrm{c}}-0.20\right) \mathrm{eV}$ and divacancies $\left(E_{\mathrm{c}}-\right.$ $0.26) \mathrm{eV}$ in the melted samples $n$-Si-Cz; divacancies and/or $\mathrm{P}_{s}-\mathrm{C}_{i}$ complexes $\left(E_{\mathrm{c}}-0.23\right) \mathrm{eV}$ in the floating zone samples $n$-Si-FZ - are acoustoactive [4,5]. It has been established that location of US induced changes is defined both by the RD structure in the sample after irradiation and electronic state of the corresponding electroactive centre. It is noteworthy that the Hall measurements of EPh characteristics in the papers $[4,5]$ were performed only in one direction of temperature changes - when heating from 100 to $300 \mathrm{~K}$. At the same time, in cyclic investigations of the temperature dependences for the charge carrier concentration $n(T)$ - both when cooling the sample $n(T \downarrow)$ and heating it $n(T \uparrow)$ - there revealed was a specific divergence (hysteresis) of temperature characteristics $\Delta n(T)=n(T \downarrow)-n(T \uparrow)$ [6]. The found divergence $\Delta n(T)$ considerably exceeds experimental errors and is not observed in respective initial unirradiated samples. Similar hysteresis $n(T)$ in annealed silicon samples $\left(5\right.$ hours at $400^{\circ} \mathrm{C}$ ) was related in the paper [7] with formation of centres characterized by a considerable time of establishment of thermodynamic equilibrium distribution over various charge states, which results in appearance of residual conductivity.

In this work, for further ascertaining the mechanism of ultrasound influence on the radiation complexes, we performed experimental investigations of EPh characteristics of $\gamma$-irradiated silicon samples $n$-Si$\mathrm{Fz}$ in different regimes, namely: stationary, after US treatment (UST), and dynamical, in-situ US loading (USL). The goal of the paper is to study and analyze the mechanism of UST and USL influence on the temperature hysteresis of charge carrier concentration $n(T)$ in the silicon crystals $n-\mathrm{Si}-\mathrm{Fz}$.

\section{Experimental}

For investigations, we chose $n$-type dislocationless silicon crystals grown using the zone melting method $n$ $\mathrm{Si}-\mathrm{Fz}$, with electron concentration $n_{300 \mathrm{~K}}=$ $(4.4 \pm 0.3) \cdot 10^{13} \mathrm{~cm}^{-3}, \quad$ specific resistance $\rho_{300 \mathrm{~K}}=$ $140 \mathrm{Ohm} \cdot \mathrm{cm}$ and background impurities $N_{\mathrm{O}}<$ $5.0 \cdot 10^{15} \mathrm{~cm}^{-3}$ and $N_{\mathrm{C}} \approx 1.0 \cdot 10^{16} \mathrm{~cm}^{-3}$. Samples were 
irradiated by $\gamma$-quanta of ${ }^{60} \mathrm{Co}$ with the dose $D \sim 10^{8} \mathrm{rad}$ $\approx 1.93 \cdot 10^{17} \gamma / \mathrm{cm}^{2}$ under room temperature, and then isochronically annealed to $T=280{ }^{\circ} \mathrm{C}$ (with a step $40{ }^{\circ} \mathrm{C}$, duration $20 \mathrm{~min}$ ). A choice was validated for the annealed samples $\left(T_{\mathrm{ann}}=280^{\circ} \mathrm{C}\right)$, in comparison with unannealed samples, effective hysteresis square $\Delta n(T)$ is a little increased (Fig. 1, curves 2, 3).

Our measurements of $n(T)$ and $\mu(T)$ were performed on the crystallographically-orientated samples with dimensions $0.7 \times 2.0 \times 9.3 \mathrm{~mm}$ by using the Hall effect method in the direct current regime (I \| [110]) and constant magnetic field $0.45 \mathrm{Tl}$ (B || [110]) directed along the perpendicular to the sample. In consecutive order, the initial samples were investigated after $\gamma$-irradiation both before and after UST, as well as during USL processing within the temperature range 100-300 K. Used longitudinal acoustic waves were radiated along the normal to the sample lengthwise to the magnetic field $B$ (frequency $f=5-15 \mathrm{MHz}$, intensity $W \leq 0.1-$ $2 \mathrm{~W} / \mathrm{cm}^{2}$, UST duration $t \approx 10^{4} \mathrm{~s}$ ). The rate of temperature changes both when cooling and heating the samples was approximately equal to $\sim 3 \mathrm{~K} / \mathrm{min}$.

\section{Results}

In the initial samples, the dependence $n(T)$ indicates full ionization of shallow donors - doping phosphorus impurity within the temperature range 100 to $300 \mathrm{~K}, n \approx$ $4 \cdot 10^{13} \mathrm{~cm}^{-3}$ (Fig. 1, curve 1). In the irradiated samples, when the temperature slows down $n(T \downarrow)$ sharply decreases, which is related with the acceptor-type RDs that freeze out the free charge carriers. Moreover, the characteristic slope of the function $\lg n(1 / T)$ for two directions of temperature changes differs, as a result the certain temperature hysteresis $n(T)$ appears. This can mean that the destiny of electroactive defects in a definite charge state at the same temperature $T_{i}<200 \mathrm{~K}$, but for different directions of temperature changes cooling and heating, respectively, is distinct. After UST of samples, one can observe the following change of the $n(T)$ shape: curve $n(T \downarrow)$ is shifted a little, becomes more steep and approaches to $n(T \uparrow)$, that after UST didn't change practically, as a result $\Delta n(T)$ is narrowed a little (Fig. 1, curves 4, 5). After continuous relaxation during several days, the dependences $n(T)$ return to the preliminary state that took place before UST (Fig. 1, curves 2,3). It should be emphasized here that similar UST of the initial unirrradiated samples (Fig. 1, curve 1) not containing any metastable RDs didn't lead to noticeable changes in $n(T)$. In contrast to UST influence, in the presence of dynamic USL the $n(T)$ hysteresis was increased (Fig. 2, curves 6,7), and when US is turned off the $n(T)$ value "momentary" (quicker than the next measurement duration - several seconds) returns to the initial state (Fig. 2, curves 2,3). It is obvious that the direction of temperature changes plays an important role: the largest acoustostimulated (AS) changes of $n(T)$ happen when cooling the sample, while when heating these are hardly observed.

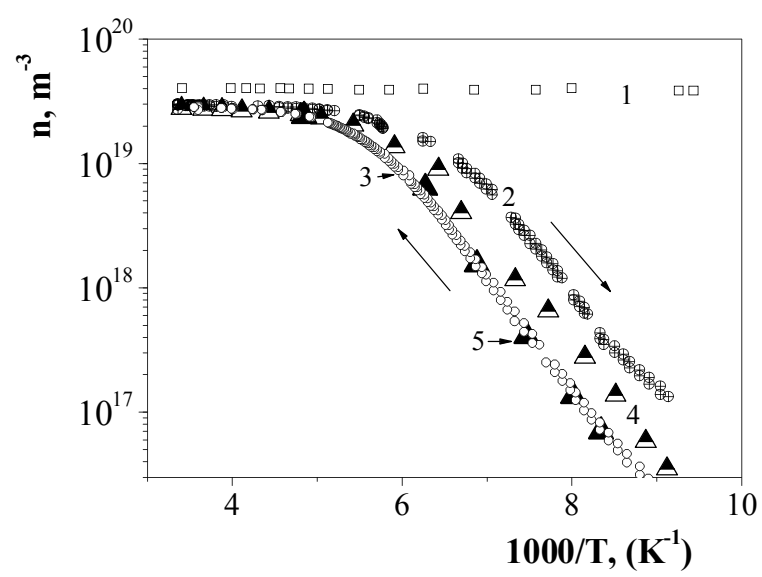

Fig. 1. Temperature dependence of the free electron concentration in $\gamma$-irradiated thermally treated silicon samples $n$-Si-FZ after US treatment. 1 - initial state of the samples before irradiation and treatments; 2,3-after $\gamma$-irradiation and thermal annealing at $280{ }^{\circ} \mathrm{C} ; 4,5-$ after $\gamma$-irradiation and annealing at $280^{\circ} \mathrm{C}$ and after UST; 2, 4- when cooling, 3, 5 when heating. Arrows point the direction of temperature changes.

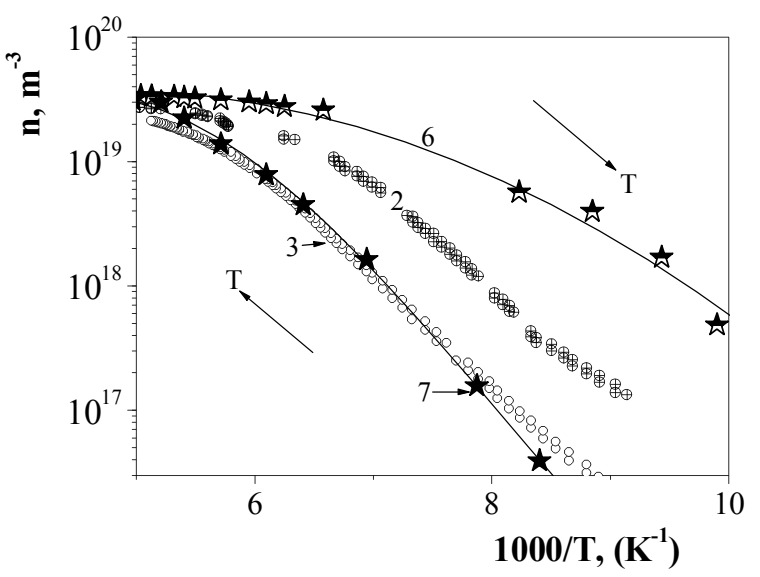

Fig. 2. Temperature dependence of the free electron concentration in $\gamma$-irradiated thermally treated silicon samples $n$-Si-FZ in the US loading regime. 2, 3 - after $\gamma$-irradiation and thermal annealing at $280{ }^{\circ} \mathrm{C} ; 6,7-$ after $\gamma$-irradiation and annealing at $280{ }^{\circ} \mathrm{C}$ and in the US loading regime; 2, 6 - when cooling; 3, 7-when heating.

\section{Discussion}

The analogous hysteresis of $n(T)$ that was observed in this work in $\gamma$-irradiated samples, in the paper [7] for the thermally treated samples was related with the structural reconstruction of the thermodonor TD- $1\left(E_{c}-0.23 \mathrm{eV}\right)$, as a result of the thermostimulated joining of an additional oxygen atom (molecule). It should be noted that at making use of annealing regime of our samples (low values of duration and annealing temperature), as known, the thermodonors is not created yet [8]. Thus, another mechanisms and defects are dominant for $\Delta n(T)$.

To explain the hysteresis effect $n(T)$, one needs to take into consideration that electroconductivity of the 
investigated samples is determined by electroactive defects manifesting the metastable character. The position of such a defect in the crystal lattice is especially determined by the sample temperature and deformation fields formed by background impurities of surroundings and/or external influence, for example by US action. And transformation into another metastable state happens via some jump [9]. Besides, the change of defect position in crystalline lattice is accompanied by the corresponding change of the charge state (Jan-Teller effect) and the corresponding change in the centre activation energy $E_{a}[10,11]$. Let us analyze which defects would be responsible for the hysteresis $n(T)$. In accord with the literature data $[9,12-14]$, in $n$-Si-Fz samples processed in the used $\gamma$-irradiation regime, the main RDs are as follows:

1. ' $\mathrm{P}_{s}-V$ ' $-E$-centres forming the level $\left(E_{c}-0.4 \mathrm{eV}\right)$ with the concentration $N_{E} \geq 10^{13} \mathrm{~cm}^{-3}$. Taking into consideration the investigated temperature range and the defect energetics, one can assume that these centres were not displayed in our experiments.

2. " $\mathrm{P}_{s}-\mathrm{C}_{i}$ " - centres in silicon with a low carbon content and high phosphorus content form in the forbidden band such levels $(0.30,0.29,0.23,0.21 \mathrm{eV})$ $[9,12-14]$. The levels $\left(E_{c}-0.23\right),\left(E_{c}-0.21\right) \mathrm{eV}$ agree with $n(T)$ experimental dependences slope. However, taking into consideration that $N_{\mathrm{C}}>>N_{\mathrm{P}}$ in our samples, the probability of $\mathrm{P}_{s}-\mathrm{C}_{i}$-centres formation is quite low, therefore, it seems that these centres are hardly responsible for AS hysteresis of $n(T)$.

3. " $\mathrm{C}_{s}-\mathrm{C}_{i}$ " - centres. At a sufficiently high carbon concentration, formation of the bistable defects $\mathrm{C}_{s}-\mathrm{C}_{i}$ with a proper level $\left(E_{c}-0.16\right) \mathrm{eV}$ is possible [9, 12-14]. In this case, the nature of bistability is determined by a weak bond between $\mathrm{C}_{i}$ and $\mathrm{C}_{s}$ atoms and possible migration of the $C_{i}$ atom at high temperatures [13]. Estimations of the possible concentration for levels and their energies allow contribution to AS effects.

4. " $V$-O" $-A$-centres are also present in the initial (after irradiation) samples. Our estimations show that for $n$-Si-Fz with $N_{\mathrm{O}}<5.0 \cdot 10^{15} \mathrm{~cm}^{-3}$ their concentration $N_{A} \leq$ $10^{12} \mathrm{~cm}^{-3}$, and with lowering the temperature down to the values $T<150 \mathrm{~K}$ their contribution to $n(T)$ is increased. However, formation of these centres is more probable in the samples with the high oxygen content grown using the Czochralsky method.

5. Divacancy $V_{2}$-centres are considered as the main RDs that define the main electrophysical and optical properties in $\gamma$-irradiated silicon samples. Their concentration $N_{V_{2}} \geq 10^{13} \mathrm{~cm}^{-3}$ [15], and they can be in different dimensional and charge states. At room temperature, the divacancy has one negative charge $\left(V_{2}^{-}\right)$, and with following temperature lowering to nitrogen ones $T<150 \mathrm{~K}$, as a result of trapping the free electrons, they acquire double negative charge $V_{2}^{2-}$. As it is known, $V_{2}{ }^{2-}$ is characterized by the level $E_{\mathrm{c}}-(0.21 \ldots 0.23) \mathrm{eV}$ in the forbidden band $[12,13]$, which corresponds to the conditions of our experiment. Divacancies $V_{2}$ can be the main defects responsible for the hysteresis effect $n(T)$, besides, moreover at the annealing temperature $280{ }^{\circ} \mathrm{C}$ they are in the unstable state. Therefore, like to $[4,5]$, we consider divacancies as the most probable acoustoactive centres.

Certainly, when the centre recharges, in particular at the temperature change of the sample, the energy level of the defect in the band $E_{R D}$ is changed discretically. Nevertheless, in the transitional region in some temperature range, while simultaneous statistical distribution of the defects over the different states is present, the smooth slope change of $n(T)$ can be observed in experiments, too. Another example of "smooth" change of $E_{R D}$ that is possible in "special" conditions arises when changing the short distance atom surroundings of the defect [16]. Diffusion approaching the background impurity atom (oxygen, carbon, nitrogen) to $\mathrm{RD}$ is caused by local deformation disfiguration of the crystal lattice and is accompanied with disturbance of the defect energy level in the forbidden band. The differences between UST and USL influences on $n(T)$ (Figs 1 and 2, respectively) indicate various mechanisms of the AS transformation of the crystal radiation defects.

Indeed, UST is the highly intensive treatment of samples $\left(W \approx 2 \mathrm{~W} / \mathrm{cm}^{2}\right)$ by means of US oscillations, which takes place at room temperature (approximately $320-330 \mathrm{~K}$ ). The US role becomes comprehensible as the stimulated factor intensifying the deformation gradient in the vicinity of the vacancy complex, on the one hand, and increases the diffusion mobility of impurity atoms, on the other hand. As a result of this "temporary approaching-joining" of impurity atoms from some surroundings, the part of divacancies is transformed in the divacancy complexes $V_{2} \rightarrow V_{2}{ }^{2-}-\mathrm{X}$ (where $\mathrm{X}$ is $\mathrm{N}, \mathrm{O}$, or $\mathrm{C}$ ); herewith their energy level is given place into the forbidden band. In particular, joining carbon to the divacancy shifts up the acceptor level energy by the value $\Delta E_{1}=0.035 \mathrm{eV}$ [17].

At the same time, the USL influence is investigated when measuring the $\mathrm{EPh}$ characteristics within the whole temperature range $(250$ to $100 \mathrm{~K})$ and at considerably lower US powers $W<0.2 \mathrm{~W} / \mathrm{cm}^{2}$. Under these conditions, the AS efficiency of the diffusion processes is already considerably reduced. In our opinion, another AS processes are dominant in this case, namely, AS acceleration of RDs transition from one metastable state to another one. Really, the position of metastable defects, that are stabilized by background impurities in surrounding crystal lattice, in the presence of USL influence becomes unstable. As a result of compression-extension of the crystal lattice by deformation fields of the acoustic wave, some distortion of the crystal cubic symmetry occurs. It results in reconstruction and orientation redistribution of $\mathrm{RD}$ dipoles, and, consequently, to additional perturbation of the centre energy position. USL in the cooling regime leads to additional ionization $V_{2}^{2-}-\mathrm{X}$, which causes the acceptor RD level to be emptied, and the charge carrier concentration inside the conduction band increases. When cooling under USL conditions, the acoustic wave 
delays freezing the carriers, which keeps a little higher electron concentration inside the conduction band, herewith the acceptor level remains less ionized (with a low quantity of charge carriers), and the mobility remains some higher due to less scattering by defects. In the case of acoustic heating the sample, contrariwise, $\mu$ would be less than without USL. When heating, USL accelerates thermal activation of carriers from acceptor levels and increases $n$ in the band.

The obtained results affirm that US actively affect on the RD state in $n-\mathrm{Si}-\mathrm{Fz}$. It is related with transformation of the defect centres due to elastic perturbation of their energy position. Moreover, UST and USL actions on RDs provide different tendencies in $\mathrm{EPh}$ parameter changes - narrowing the temperature hysteresis $n(T)$ at UST and their widening at USL, and it is defined by the initial (on the moment of the US action) state of the metastable defects.

\section{Conclusions}

Thus, to explain the differences between impacts of US treatment and US loading on the EPh characteristics of $n$-Si-Fz, we suppose that: a) US treatment of long duration for silicon samples at high temperatures $(>320 \mathrm{~K})$ results in residual diffusion modification of the impurity-defect crystal system, which is caused by perturbation of the RD level energy position (divacancy, $\mathrm{P}_{s}-\mathrm{C}_{i}$ centre) inside the band in the consequence of temporary approaching-capture of a background impurity atom (oxygen, carbon, nitrogen). It corresponds to the Jellison model and could be related with reverse relaxation of the non-equilibrium conduction in the result of internal reverse defect modification; b) US dynamic influence at low temperatures $(<200 \mathrm{~K})$ is reversible and caused by AS deformation change in the metastable defect concentration distribution between different configuration and energy states as well as respective distribution of the carrier concentration over these levels.

\section{References}

1. O.Ya. Olikh and I.V. Ostrovsky, Increase of electron diffusion length in $\mathrm{p}$-Si crystals under ultrasound action // Fizika tverdogo tela 44 (7), p. 1198-1202 (2002), in Russian.

2. A.A. Podolyan, V.I. Khivrych, Ultrasound influence on the radiation defects treatment in silicon at the room temperatures // Technical Physics Letters 31 (10), p. 11-16 (2005), in Russian.

3. A.P. Onanko, A.A. Podolyan, I.V. Ostrovsky, Ultrasound treatment influence on the internal friction in silicon // Technical Physics Letters 29 (15), p. 40-44 (2003), in Russian.

4. Ya.M. Olikh, N.D. Timochko, A.P. Dolgolenko, Acoustic-wave-stimulated transformations of radiation defects in $\gamma$-irradiated $n$-type silicon crystals // Technical Physics Letters 32 (13), p. 6773 (2006), in Russian.

5. V.M. Babych, O.P. Dolgolenko, Ya.M. Olikh, M.D. Tymochko, Ultrasound influence on the electrical activity of radiation defects in $\gamma$-irradiated n-type silicon crystals // Nuclear Physics and Atomic Energy 1 (19), p. 95-102 (2007), in Ukrainian.

6. V.M. Babych, Ya.M. Olikh, M.D. Tymochko, About differences of stationary and dynamic ultrasound on electrical activity of radiation defects in $\gamma$-irradiated $n$-type silicon crystals obtained by zone melting // 6-th Intern. Confer."Actual Problems of Semiconductor Physics", Drogobych University Publ., September 23-26, 2008, p. 177 (in Ukrainian).

7. L.F. Makarenko, V.P. Markevych, L.I. Murin, Reconstructed thermodonors in silicon - defects with $U<0$ // Fizika i tekhnika poluprovodnikov 19 (11), p. 1935-1939 (1985) (in Russian).

8. V.M. Babych, M.I. Bletskan, E.F. Venger, Oxygen in Silicon Monocrystals. Interpress LTD, Kyiv, 1997 (in Russian).

9. B.N. Mukashev, Kh.A. Abdullin, Yu.V. Gorelkinsky, Metastable and bistable defects in silicon // Uspekhi fizicheskikh nauk 170 (2), p. 143-155 (2000), in Russian.

10. S.S. Moliver, Method of an open envelope for the electronic structure of silicon divacancy // Fizika tverdogo tela 41 (3), p. 404-410 (1999), in Russian.

11. J. Bourgoin, M. Lannoo, Point Defects in Semiconductors II. Experimental Aspects. Mir, Moscow, 1985 (in Russian).

12. M.T. Asom, J.L. Benton, R. Sauer, and L.C. Kimerling, Interstitial defect reactions in silicon // Appl. Phys. Lett. 51 (4), p. 256-258 (1987).

13. L.W. Song, B.W. Benson, and G.D. Watkins, Identification of a bistable in silicon: The carbon interstitial-carbon subsitutional pair // Appl. Phys. Lett. 51 (15), p. 1155-1157 (1987).

14. V.S. Vavilov, V.F. Kiselev, B.N. Mukashev, Defects in Silicon and on its Surface. Nauka Publ., Moscow, 1990 (in Russian).

15. I.D. Konozenko, A.K. Semenjuk, V.I. Khivrych, Radiation Effects in Silicon. Naukova dumka, Kyiv, 1974 (in Russian).

16. G.E. Jellison, Transient capacitance studies of an electron trap at $\mathrm{E}_{\mathrm{C}}-\mathrm{E}_{\mathrm{T}}=0.105 \mathrm{eV}$ in phosphorusdoped silicon // J. Appl. Phys. 53 (8), p. 5715-5719 (1982).

17. A.P. Dolgolenko, P.G. Lytovchenko, M.D. Varentsov, G.P. Gaidar, A.P. Lytovchenko, Peculiarity of radiation defects creation in silicon with a low and high free oxygen concentration // Zbirnyk naukovykh prats' institutu yadernykh doslidzhen' No. 2(15), p. 106-114 (2003), in Russian. 